\title{
La singulière bonne santé des indépendants
}

The singular good health of self-employed workers

\section{Élisabeth Algava, Catherine Cavalin et Sylvie Célérier}

\section{CpenEdition}

\section{Journals}

Édition électronique

URL : http://journals.openedition.org/travailemploi/5806

DOI : 10.4000/travailemploi.5806

ISSN : 1775-416X

\section{Éditeur}

DARES - Ministère du Travail

\section{Édition imprimée}

Date de publication : 15 décembre 2012

Pagination : $5-20$

ISSN : 0224-4365

\section{Référence électronique}

Élisabeth Algava, Catherine Cavalin et Sylvie Célérier, "La singulière bonne santé des indépendants », Travail et Emploi [En ligne], 132 | octobre-décembre 2012, mis en ligne le 01 décembre 2014, consulté le 30 avril 2019. URL : http://journals.openedition.org/travailemploi/5806 ; DOI : 10.4000/ travailemploi.5806 


\title{
La singulière bonne santé des indépendants
}

\author{
Élisabeth Algava ${ }^{(*)}$, Catherine Cavalin ${ }^{(* *)}$, Sylvie Célérier ${ }^{(* *)}$
}

\begin{abstract}
Le statut d'indépendant abrite des situations que l'on sait très hétérogènes. Les travailleurs concernés semblent ne partager qu'un même profil de temps de travail marqué par de nombreuses heures, une forte variabilité et une relative autonomie de décision en la matière. L'article propose de suivre la piste d'une autre caractéristique commune, relative à leur état de santé que les indépendants déclarent meilleur que les salariés bien qu'ils soient un peu plus âgés en moyenne. Cette hypothèse est testée sous plusieurs angles et validée en écartant notamment l'éventuel jeu des catégories socioprofessionnelles derrière les différences de statuts. Sont également comparées les suites de la maladie sur l'activité professionnelle selon les différentes situations professionnelles retenues. Les résultats tout à la fois confirment le meilleur état de santé relatif des indépendants et suggèrent un mode singulier d'articulation entre la santé et le travail justifiant la poursuite des travaux sur la santé de ce groupe particulier pour en comprendre les spécificités et pour compléter nos connaissances sur les mécanismes généraux par lesquels la santé et le travail se font constamment écho.
\end{abstract}

L'intérêt des chercheurs pour le groupe des travailleurs indépendants a été récemment réactivé par la création du nouveau statut d'auto-entrepreneur ${ }^{(1)}$. Le succès de ce dernier a prolongé les réflexions critiques sur les solutions proposées au chômage et la dérégulation dont elles peuvent être la cause. Le nouveau statut est ainsi soupçonné de transférer vers les individus les risques de l'activité productive sans que ces derniers y trouvent beaucoup de gain ${ }^{(2)}$. Hors cet intérêt récent, la situation des indépendants reste plus rarement explorée. Les frontières de ce groupe posent, il est vrai, de délicates questions dont les auteurs font état dès les premières lignes de leurs textes. Y sont regroupées en effet une variété de situations professionnelles à la fois très typées et distinctes entre elles, qu'il s'agisse de la nature de l'activité, des catégories socioprofessionnelles impliquées, du statut d'employeur ou d'indépendant isolé( ${ }^{(3)}$, du type et du montant des revenus ou encore du régime assurantiel. Ajoutons que les explorations statistiques se heurtent rapidement à des effectifs qui ne sont pas toujours suffisants pour conduire des analyses détaillées. Le statut juridique d'indépendant ne peut donc prétendre désigner une

(*) Ministère du Travail, de l'Emploi, de la Formation professionnelle et du Dialogue social, Direction de l'animation de la recherche, des études et des statistiques (Dares); elisabeth. algava@dares.travail.gouv.fr

$(* *)$ Centre d'études européennes de Sciences Po, Centre d'études de l'emploi; catherine.cavalin@sciences-po.fr

(***) Centre d'études de l'emploi, Centre Pierre Naville-université d'Evry-Val-d'Essonne; sylvie.celerier@cee-recherche.fr

(1) Loi du 4 août 2008 de modernisation de l'économie, n $2008-776, J O, 5$ août 2008, p. 1271.

(2) Voir par exemple : Levratto, Serverin (2009); Domens, Pignier (2012).

(3) En 2005, $51 \%$ d'indépendants travaillaient seuls, $40 \%$ étaient employeurs et $9 \%$ aides familiaux (AlgaVA, VINCK, 2009). réalité stabilisée de l'emploi ou du travail. Il invite à la plus grande prudence dès qu'il s'agit de chercher des traits qui seraient communs à l'ensemble de ses composantes.

La littérature reconnaît pourtant aux indépendants une particularité commune, qu'elle constate d'ailleurs plus qu'elle ne l'explore vraiment. Elle concerne leur temps de travail considéré du point de vue de l'organisation temporelle de leurs activités professionnelles et de la maîtrise qu'ils déclarent en avoir. Nous proposons dans cet article d'argumenter l'hypothèse d'un autre trait partagé, portant cette fois sur leur état de santé, précisément leur meilleur état de santé relativement aux salariés. Cette position apparemment plus favorable des indépendants a déjà été notée dans la littérature et en partie discutée ${ }^{(4)}$. Mais ces analyses s'en tiennent à un nombre restreint d'indicateurs, extraits parfois de bases non représentatives de la population générale ${ }^{(5)}$. La comparaison avec les salariés y est par ailleurs menée de façon globale quand la diversité des situations du statut appellerait une différenciation par sous-groupe. La comparaison des conditions de travail entre salariés et non-salariés à partir des données de l'enquête Conditions de travail 2005 (Dares) par exemple montre l'intérêt d'une telle différenciation. Ses résultats soulignent en effet les proximités des indépendants et des salariés engagés dans les mêmes domaines d'activité, notamment les agriculteurs exploitants et les artisans d'un côté et

(4) Par exemple : InSERm (2011), chapitre 5 : «État de santé des travailleurs indépendants selon le secteur professionnel», pp. 83-977; Ha-VINH et al. (2010a); SAUZE et al. (2008).

(5) Par exemple les fichiers des Affections de longue durée (ALD). 
les ouvriers de l'autre (AlgaVA, VINCK, 2009). Ils témoignent ainsi du caractère second du statut.

Si elle s'avérait, l'hypothèse d'une corrélation entre le statut d'indépendant et leur meilleur état de santé relatif donnerait l'indice d'un mode d'articulation singulier entre le travail et la santé qu'il serait intéressant d'explorer. En effet, rien ne laisse prévoir un tel résultat, d'autant que l'on sait les indépendants plus âgés en moyenne que les salariés. Se poserait alors la question des mécanismes susceptibles d'expliquer cette singularité. Le premier auquel on pense - celui qui motive principalement nos investigations - se rapporte aux systèmes d'assurance sociale attachés au statut d'indépendant que l'on sait différents de ceux des salariés ${ }^{(6)}$. Les déclarations de santé des indépendants donnent précisément l'occasion d'explorer cet aspect rarement considéré dans la compréhension des liens entre santé et travail, mais très certainement structurant. De façon en partie liée, nous pourrions également suivre la piste d'une spécificité de déclaration de santé ou de comportements de soin de ce groupe comme certains travaux récents le suggèrent (Amossé et al., 2012). Bref, un programme de recherches s'annonce qui exige cependant que sa première étape, c'est-à-dire la réalité de la meilleure santé relative des indépendants, soit établie aussi rigoureusement que possible. Nous y consacrons une partie de cet article.

L'hypothèse d'une meilleure santé relative des indépendants est testée sur la base d'enquêtes statistiques réalisées auprès d'échantillons représentatifs de la population générale qui offrent par ailleurs un éventail élargi d'indicateurs de santé. La santé dont nous traitons ici est construite par ces indicateurs de santé. Elle est déclarée par les travailleurs et non inférée des caractéristiques de leurs conditions de travail(7). Précisément, nous avons mobilisé : l'enquête Handicap Santé - volet Ménages de 2008 (HSM) pilotée par la Drees et l'Insee ${ }^{(8)}$ et réalisée en 2008 auprès de 39000 personnes, dont 931 nonsalariés actifs en emploi (9), ainsi que l'enquête Santé et itinéraire professionnel (SIP) de 2006, menée par la Drees et la Dares auprès de 14000 individus comptant 879 non-salariés actifs en emploi. Ces deux enquêtes offrent une richesse d'indicateurs très satisfaisante pour notre projet. La taille de leurs échantillons respectifs permet en outre de détailler

(6) Pour une analyse détaillée de ces différences, se reporter à Algava, Cavalin, Célérier (2011).

(7) Pour un argumentaire détaillé de cette option, voir CAVALIN, CÉlÉrier (2012a) et pour un examen des différentes voies de compréhension des liens entre santé et travail, voir CAVALIN, CÉlÉRIER (2012b).

(8) Drees : Direction de la recherche, des études, de l'évaluation et des statistiques; Insee : Institut national de la statistique et des études économiques.

(9) Cette enquête associe les thèmes de la santé et du handicap et prolonge l'enquête décennale Santé 2003 et Handicaps Incapacités Dépendance (HID, 1998-2001). suffisamment les différentes populations sans que la robustesse statistique des résultats ne s'en trouve trop vite mise en cause.

L'exercice a visé - pour les écarter - trois principaux mécanismes statistiques susceptibles de construire une corrélation artificielle entre statut d'emploi et état de santé. D'abord, les effets structurels d'autres variables tels l'âge et le sexe que l'on sait décisives dans les déclarations d'état de santé et qui concernent directement les indépendants qui sont plus âgés en moyenne que les salariés et plus souvent des hommes. Ensuite, le jeu possible du gradient social(10) à l'intérieur du groupe statutaire - comme on en connaît pour les salariés entre les cadres et les ouvriers - qui distinguerait favorablement les professions libérales indépendantes. Les solides acquis de la littérature sur ce thème(11) invitent à prendre en compte la catégorie socioprofessionnelle des personnes (PCS) complétée, aujourd'hui, par d'autres caractéristiques sociales des individus comme le revenu (lui-même lié à la PCS), le diplôme ou le statut d'assuré social (TuBeuf, 2010) ${ }^{(12)}$ qui semblent structurer les déclarations de santé et les comportements de soins. Enfin, dernier mécanisme à envisager : une possible sélection par la santé(13) à l'entrée ou en sortie du statut, telle qu'il en existe pour le travail de nuit.

Nos analyses confirment et précisent l'avantage relatif des indépendants en matière de santé que l'on peut donc considérer comme suffisamment robuste pour y asseoir le programme de recherche envisagé qui en approfondira la compréhension. Ces analyses récusent l'hypothèse d'effets structurels indirects ou d'une compensation interne entre sous-groupes telle que la littérature sur les inégalités sociales de santé invite à la chercher. Quand elles existent, les inégalités entre d'un côté les agriculteurs, commerçants et artisans et de l'autre, les professions libérales ne sont jamais si complètes, ni

(10) Cette notion renvoie à ce que l'on désigne également par inégalités sociales de santé. L'Organisation mondiale de la santé $(\mathrm{OMS})$ la définit en référence à un résultat régulier et général : "À l'intérieur des pays, les données montrent qu'en général, plus un individu occupe une position socio-économique défavorable, plus il est en mauvaise santé : ce gradient social concerne toute l'échelle socio-économique, de haut en bas. Il s'agit d'un phénomène mondial, que l'on constate dans les pays à revenu faible ou intermédiaire comme dans ceux à revenu élevé. Le gradient social signifie que les inégalités sanitaires touchent tout un chacun». Pour la définition complète du concept se reporter au site de l'OMS : http://www.who.int/ social_determinants/final_report/key_concepts/fr/

(11) Notamment CAmbors et al. (2008) pour l'espérance de vie et l'espérance de vie sans limitation et, plus largement sur le thème, voir la bibliographie complète établie par l'Institut de recherche et documentation en économie de la santé (Irdes) en 2012 et disponible à cette adresse : http://www.irdes.fr/ EspaceDoc/DossiersBiblios/InegalitesSocialesSante.pdf; voir également BEH (2011).

(12) Voir également Chauvel (2001) pour la complexité de l'approche des «classes sociales».

(13) Dite encore "effet du travailleur en bonne santé» ou «Healthy Worker Effect». 
ne jouent toujours dans le même sens, que celles qui s'observent entre cadres et ouvriers salariés. Cette non-conformité à un résultat maintes fois et partout observé est un fruit inattendu de nos investigations qui confirme l'intérêt de la question posée et dont, pour l'heure, nous exposons précisément les termes. Reste l'éventualité d'une sélection par la santé que l'on ne peut écarter aussi fermement en l'absence de données longitudinales ${ }^{(14)}$.

Les enquêtes transversales disponibles ne sont cependant pas dénuées de ressources et nous avons mobilisé les données rétrospectives de l'enquête SIP sollicitant la mémoire des enquêtés sur leur itinéraire professionnel et des problèmes de santé qu'ils disent avoir rencontrés. Ces données décrivent un léger mouvement du non-salariat vers le salariat à l'occasion de la survenue de problèmes de santé, mais rien d'assez net cependant pour démontrer un effet travailleur sain. Nos effectifs et l'ampleur du mouvement restent faibles en effet et la mémoire des enquêtés, peut-être, fragile. Cependant, les tentatives en ce sens sont loin d'avoir été inutiles. Elles ont invité à suivre les effets comparés selon le statut de la survenue de problèmes de santé sur le déroulement de l'itinéraire professionnel et à considérer certains événements tels l'arrêt maladie ou la modulation des heures de travail. Ces événements retrouvent certaines des spécificités du statut d'indépendant, que ce soit du point de vue du type de travail effectué (horaires) que des caractéristiques de leurs systèmes d'assurance (arrêt maladie). Interrogeant l'éventualité d'un effet travailleur sain, nous nous sommes donc d'ores et déjà engagées dans la réflexion sur les sources de l'avantage de santé constaté.

Présentant l'ensemble de ces analyses, notre propos se partage en deux temps relativement autonomes quant aux questions traitées et aux ressources mobilisées. Le premier s'attache au constat statistique de la meilleure santé relative des indépendants dont il tente d'apporter la preuve en faisant la part des effets de structure et du jeu des inégalités sociales de santé. Nous y rappelons également quelques dimensions-clefs de l'organisation temporelle de l'activité indépendante qui spécifient le groupe et sont susceptibles de peser comme des contraintes pour la santé. Le second temps présente les suites de la réflexion sur l'effet travailleur sain et compare les situations des indépendants et des salariés quand la maladie survient. Nous y suivons les effets de la maladie sur le cours de leur itinéraire professionnel sur plusieurs années, la nature et l'intensité de leur recours à l'arrêt maladie et l'ampleur de leur capacité à moduler leur temps de travail.

(14) La mise à disposition prochaine de l'édition 2010 de l'enquête SIP offrira cette possibilité, 11000 des 14000 répondants de 2006 ayant pu être réinterrogés.

\section{La santé des indépendants : l'autre spécificité d'un groupe hétérogène}

Groupe varié, les indépendants posent d'emblée la question de la convention à retenir pour les définir, aucune ne faisant consensus à ce jour. La plus couramment utilisée, et que nous reprendrons nous-mêmes en dépit de ses ambiguïtés, vient de la statistique publique. Elle constitue, en creux du salariat, la catégorie dite des non-salariés ${ }^{(15)}$ sans toutefois assurer une distinction parfaite du salariat. Une distinction au demeurant problématique tant la frontière entre indépendance et salariat semble parfois fragile (RolLe, 1988). La jurisprudence très active en France reconnaît ainsi régulièrement de multiples formes d'assujettissement déguisées sous une autonomie juridique affichée (MAURIN et al., 1999; SUPIOT, 2009). Réciproquement, certains groupes de salariés tels les cadres et professions intellectuelles supérieures, voire certaines des professions intermédiaires, jouissent d'une autonomie professionnelle très comparable à celle qui est censée caractériser les non-salariés. Enfin, les individus franchissent souvent eux-mêmes les frontières de statut au cours de leur carrière. L'enquête SIP 2006 nous apprend qu'ils la franchissent bien plus souvent du salariat vers l'indépendance, la plupart des non-salariés $(71 \%)$ ayant déjà eu une expérience de salarié. La situation inverse ne concerne que $5 \%$ des salariés qui déclarent être passés par le statut d'indépendant.

Sur la base de la convention statistique retenue, quelque trois millions de personnes composent le non-salariat en 2010 (16), soit plus qu'en 2009 et 2008. Ces trois millions représentaient $11 \%$ de la population active occupée, avec un déséquilibre patent - et régulier pour ce groupe - entre les hommes $(68 \%)$ et les femmes ${ }^{(17)}$. Les données rassemblées dans le tableau 1 complètent la présentation de ce groupe et témoignent encore de son hétérogénéité, présentée ici du point de vue du niveau de vie et des diplômes (voir les cases grisées pour chaque sous-catégorie) ${ }^{(18)}$.

(15) La nomenclature de l'Insee y regroupe les indépendant-e-s à leur compte, les personnes qui déclarent aider un membre de leur famille dans son travail sans être rémunérées (aides familiaux), les chefs d'entreprise non-salariés employant des salariés. La catégorie n'est pas sans ambiguïté cependant puisqu'y sont également comptés les PDG, les gérant-e-s minoritaires et les associé-e-s.

(16) Enquête Emploi en continu (EEC, Insee, 2010).

(17) Autre déséquilibre interne à la catégorie des non-salariés : les femmes représentent les trois quarts des aides familiaux tandis qu'elles comptent pour $30 \%$ des personnes à leur compte et des chefs d'entreprise (EEC, Insee, 2010).

(18) «Les informations disponibles sur les revenus des indépendants sont approximatives et difficilement exploitables» (BessiÈre et al., 2011) du fait de la variabilité annuelle des revenus des agriculteurs, du revenu que se verse ou non le non-salarié selon le réinvestissement de son bénéfice dans son activité, de la fiabilité des déclarations fiscales. La pertinence de la variable «niveau de vie», limitée (INSEE, 2009), invite à se tourner vers le patrimoine des indépendants. Mais celui-ci n'est pas accessible dans les données d'enquêtes déclaratives sur la santé ici mobilisées. 
Tableau 1 : Caractéristiques des différentes composantes des non-salariés

\begin{tabular}{|c|c|c|c|c|c|c|c|c|c|c|c|c|}
\hline & \multirow[b]{2}{*}{$\begin{array}{c}\text { Effectif } \\
\text { brut }\end{array}$} & \multicolumn{5}{|c|}{ Tranches de niveau de vie (euros) } & \multicolumn{4}{|c|}{ Diplôme le plus élevé obtenu } & \multicolumn{2}{|c|}{ Âge } \\
\hline & & $\begin{array}{c}10 \\
800[\end{array}$ & $\begin{array}{c}{[800} \\
1100[\end{array}$ & $\begin{array}{l}{[1100} \\
1500[\end{array}$ & $\begin{array}{l}{[1500} \\
2000[\end{array}$ & $\begin{array}{c}{[2000} \\
\text { et }+\end{array}$ & Brevet & $\begin{array}{l}\text { BEP } \\
\text { CAP }\end{array}$ & Bac & $\begin{array}{c}\text { Bac } \\
+2 \\
\text { ou }+\end{array}$ & moyen & médian \\
\hline Agriculteurs exploitants & 230 & $18 \%$ & $18 \%$ & $28 \%$ & $12 \%$ & $13 \%$ & $16 \%$ & $61 \%$ & $7 \%$ & $16 \%$ & 45 & 46 \\
\hline Artisans commerçants & 437 & $11 \%$ & $13 \%$ & $22 \%$ & $22 \%$ & $23 \%$ & $25 \%$ & $48 \%$ & $10 \%$ & $17 \%$ & 45 & 45 \\
\hline $\begin{array}{l}\text { Professions libérales et } \\
\text { assimilés }\end{array}$ & 146 & $4 \%$ & $4 \%$ & $8 \%$ & $13 \%$ & $62 \%$ & $5 \%$ & $8 \%$ & $6 \%$ & $81 \%$ & 46 & 47 \\
\hline «Autres» non-salariés & 103 & $5 \%$ & $8 \%$ & $13 \%$ & $33 \%$ & $30 \%$ & $7 \%$ & $14 \%$ & $14 \%$ & $65 \%$ & 44 & 44 \\
\hline Ensemble non-salariés & 931 & $11 \%$ & $12 \%$ & $20 \%$ & $19 \%$ & $27 \%$ & $18 \%$ & $40 \%$ & $9 \%$ & $33 \%$ & 45 & 45 \\
\hline Ensemble actifs occupés & 8653 & $7 \%$ & $12 \%$ & $23 \%$ & $26 \%$ & $28 \%$ & $20 \%$ & $34 \%$ & $11 \%$ & $34 \%$ & 40 & 41 \\
\hline Ensemble & 17322 & $13 \%$ & $13 \%$ & $22 \%$ & $22 \%$ & $23 \%$ & $27 \%$ & $31 \%$ & $13 \%$ & $28 \%$ & 41 & 41 \\
\hline
\end{tabular}

Lecture : $18 \%$ des agriculteurs exploitants perçoivent un revenu par unité de consommation inférieur à 800 euros tandis que $4 \%$ des professions libérales et assimilés sont dans ce cas.

Champ : Population de 18 à 65 ans résidant en France métropolitaine en ménage ordinaire.

Source : Enquête Handicap Santé Ménages, 2008, Insee-Drees.

\section{Les temps du travail}

La diversité du groupe se vérifie également du point de vue des conditions de travail des différentes composantes des non-salariés. Les analyses confirment l'importance de l'activité proprement dite sur laquelle, nous l'avons vu, ils se rapprochent bien davantage de leurs équivalents salariés que de ceux qui partagent le statut d'indépendant (Gollac, VolKoff, 2000). Une dimension cependant fait exception qui les réunit tous et les distingue des salariés. Elle concerne l'organisation temporelle de leurs activités professionnelles, du double point de vue du temps consacré au travail et de ce qui est encore appelé l'emprise du travail sur la vie(19). En effet, quelle que soit l'enquête considérée, les non-salariés déclarent toujours un plus grand nombre d'heures de travail que les salariés, y compris quand ces derniers sont cadres. La dernière mesure de l'enquête Emploi du temps (2010) enregistre ainsi un temps de travail indépendant supérieur en moyenne de $10 \mathrm{~h} 30$ par semaine à celui des salariés (RICROCH, ROUMIER, 2011) ${ }^{(20)}$.

Les données de l'enquête Emploi en continu 2010 (Insee) dessinent des profils temporels également très différents selon le statut quant aux durées déclarées et aux rythmes hebdomadaires. Tandis que les durées de travail hebdomadaires des salariés se centrent, comme attendu, autour de la durée légale, celles des non-salariés présentent une distribution beaucoup plus étalée, s'étendant jusqu'à la tranche des 60-65 heures (voir graphique). Les indépendants déclarent une variabilité plus forte de

(19) Cet indicateur rend compte d'une forte porosité entre temps de travail et temps privé. Il considère cinq traits de l'organisation du travail détaillés dans le tableau 2 qui, si trois d'entre eux sont satisfaits, apprécient «une très forte emprise du travail».

(20) Soit quatre heures d'écart de moins que lors de la précédente édition de l'enquête en 1999; réduction qui s'explique essentiellement par un plus grand nombre de jours de congé des indépendants et non par une réduction du temps de travail moyen qu'ils fournissent par semaine travaillée. leurs horaires d'une semaine à l'autre rejoignant les données de l'enquête Conditions de travail (2005) qui montraient également le plus grand nombre de jours de la semaine qu'ils consacrent au travail englobant généralement le samedi et, plus rarement, le dimanche. En ajoutant d'autres contraintes liées au temps de travail, la même enquête concluait à une emprise du travail dix fois plus fréquente pour les non-salariés que pour les salariés (voir le tableau 2). Certes, la distinction par composante des non-salariés montre des disparités - notamment entre les agriculteurs et les professions libérales mais les valeurs respectives restent supérieures à celles des salariés (21).

La régularité avec laquelle la littérature enregistre ces différences de profils horaires des salariés et des non-salariés témoigne de la robustesse de ce trait et de son caractère quasi structurel. Dans la typologie des organisations du temps de travail qu'Alain Chenu dégageait depuis l'enquête Emploi du temps 1999, l'emploi du temps des indépendants suffisait d'ailleurs à les distinguer de tous les autres enquêtés, créant à eux seuls l'un des six types identifiés (CHENu, 2002). Dans ce type, se combinaient de lourds horaires, étendus sur six jours au moins de la semaine, de fortes variations d'une semaine à l'autre, un travail au domicile plus fréquent, peu de travail de nuit, une relative autonomie d'organisation temporelle appréciée par une plus grande liberté de décider du moment des pauses et le sentiment de constamment manquer de temps ${ }^{(22)}$.
(21) À l'exception du travail de nuit, toujours inférieur.

(22) Quelques années auparavant, Nathalie MissĖGue (2000) parvenait à des résultats semblables en partant des données de l'enquête Emploi 2005 et de son module ad hoc sur le temps de travail. 


\section{Graphique : Temps et rythmes de travail}
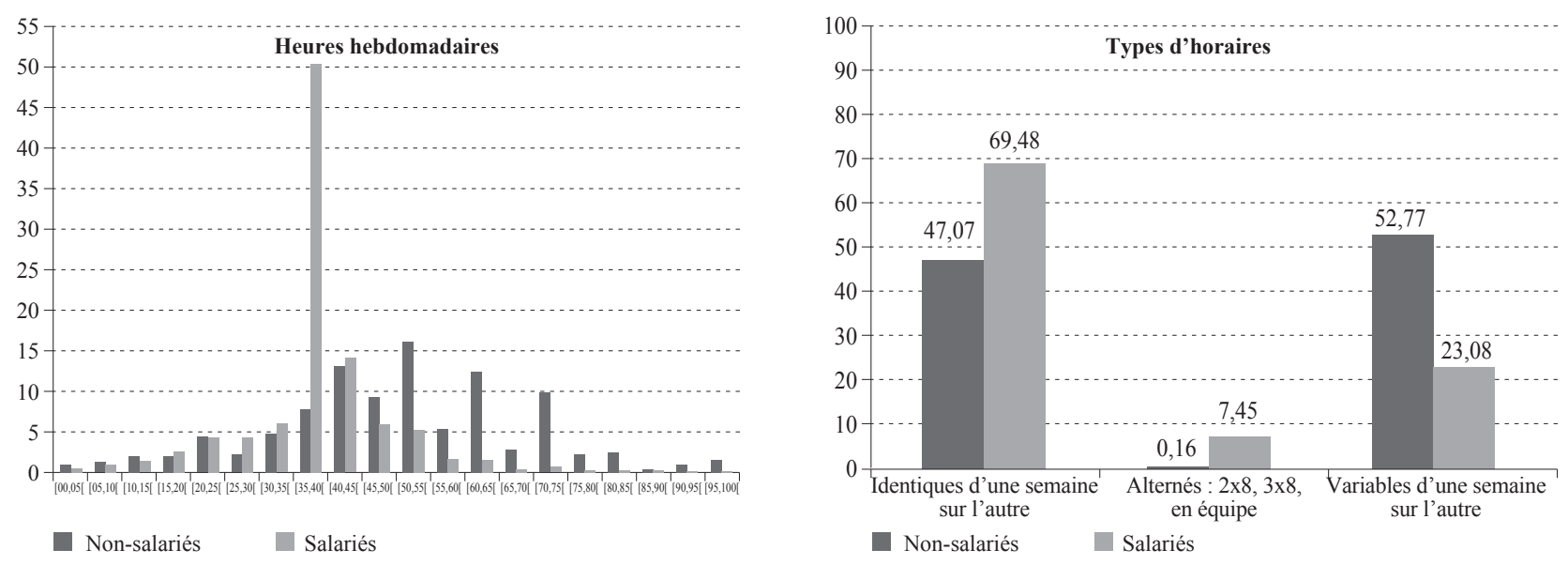

Lecture : La partie gauche du graphique compare le nombre moyen d'heures de travail par semaine dans l'emploi principal (emploi régulier) déclaré selon le statut d'emploi. La partie droite du graphique compare les types d'horaires déclarés dans la semaine et d'une semaine sur l'autre, selon le statut d'emploi.

Champ : Actifs occupés interrogés en rang d'interrogation 1 de l'Enquête emploi en continu (2010).

Source : Enquête emploi en continu (Insee, 2010).

Tableau 2 : Emprise du travail

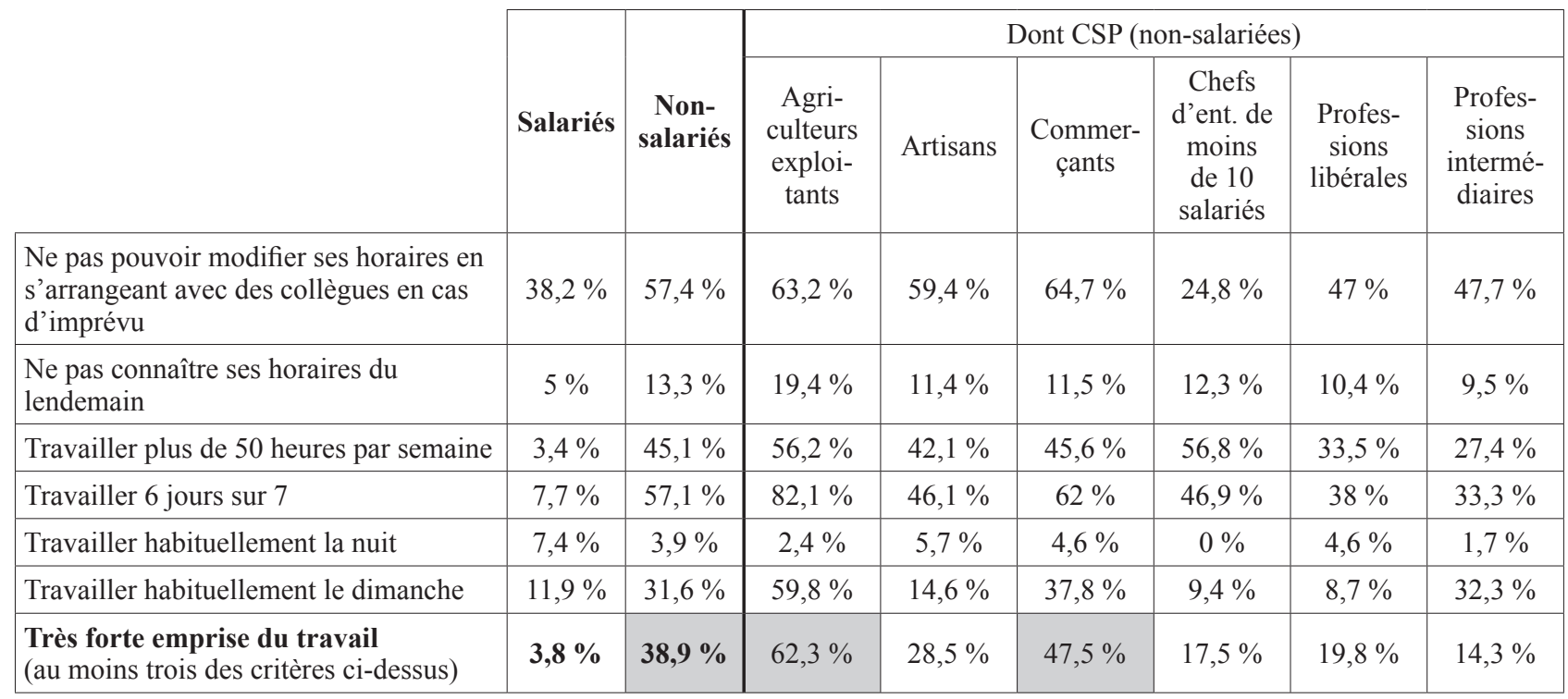

Lecture : 38,9 \% des non-salariés sont caractérisés par une très forte emprise du travail selon les trois critères mesurés par l'enquête Conditions de travail (2005).

Source : Enquête Conditions de travail (2005), repris d'AlgaVA, VINCK (2009).

\section{Des traits communs de la santé}

À ce trait distinctif des indépendants qui concerne l'organisation temporelle du travail, nous proposons d'ajouter un autre trait relatif à leurs déclarations de santé. La proposition appelle quelques précisions, car si l'on admet - parfois un peu vite - que la particularité de l'engagement des indépendants dans leur travail puisse se traduire par une charge horaire plus lourde et une plus forte porosité des différents pans de la vie, on voit moins les raisons qui les feraient partager un état de santé similaire.
À un niveau descriptif, on constate que l'âge moyen relativement élevé des non-salariés (voir tableau 1) ${ }^{(23)}$ ne semble pas peser sur leur état de santé comme on aurait pu s'y attendre. Leurs réponses aux

(23) À l'exception des agriculteurs - et encore que les choses changent, avec l'élévation de l'espérance de vie des générations les plus anciennes et le coût de plus en plus élevé de l'installation -, les indépendants commencent rarement leur carrière professionnelle sous ce statut. Ils y accèdent après des expériences salariées préalables ou, dans le cas des professions libérales, à l'issue d'études longues (EvaIn, Amar, 2006). 


\section{Encadré}

\section{Détail des sous-groupes salariés et non-salariés utilisés dans les régressions logistiques}

Dans les régressions logistiques présentées, le statut d'emploi se fonde en premier lieu, comme indiqué plus haut, sur la variable «STATUT» qui, dans les enquêtes Insee, rassemble dans les «non-salariés» les personnes «indépendantes, à leur compte" (STATUT = 5), les "aides familiaux" (STATUT = 6) et les "chefs d'entreprises salariés, PDG, gérants minoritaires ou associés" (STATUT =7). Sur la base de cette variable, les tableaux 3 et 4 distinguent nonsalariés et salariés et, au sein de ceux-ci, identifient les "cadres et professions intellectuelles supérieures" par le groupe socioprofessionnel « 3 », les autres salariés relevant de tous les autres groupes de la nomenclature des PCS. Le tableau 5 pousse plus loin la logique qui désagrège les salariés d'une part, les non-salariés de l'autre, en combinant statut d'emploi et PCS comme suit :

Non-salariés :

- Agriculteurs, commerçants et artisans : STATUT $=5,6,7$ et CS = 10, 21, 22 ( $n=667)$;

- Professions libérales, cadres et professions intellectuelles supérieures, chefs d'entreprises de 10 salariés et plus : $\operatorname{STATUT}=5,6,7$ et $\mathrm{CS}=23,31,32,36(\mathrm{n}=146)$;

- «Autres» non-salariés : STATUT $=5,6,7$ et CS $=41,46,47,48,54,55,56,61,66,69$ ( $n=103)$.

Salariés :

- Salariés cadres et professions intellectuelles supérieures : STATUT $=1,2,3,4$ et CS $=31,32,36(n=1033)$;

- Salariés professions intermédiaires : STATUT $=1,2,3,4$ et CS $=41,46,47,48(n=1789)$;

- Salariés employé-e-s et ouvriers : STATUT $=1,2,3,4$ et CS $=51,54,55,56,61,66,69$ ( $n=4784)$.

trois questions du mini-module européen de santé(24) (HSM 2008) les placent en effet dans une position intermédiaire entre les salariés cadres (les plus avantagés) et les salariés non-cadres ${ }^{(25)}$. S'appuyant sur un raisonnement à caractéristiques données qui permet de faire la part des effets de structure, le tableau 3 précise la nature de cet avantage de santé. Le raisonnement «toutes choses égales par ailleurs» intègre ici les variables âge, sexe, statut d'emploi (voir encadré), niveau de vie et couverture maladie complémentaire, c'est-à-dire le fait de disposer ou non d'une complémentaire santé.

Les non-salariés présentent une probabilité plus faible de déclarer des symptômes combinés (colonne I) et des maladies (colonne II) que les salariés non-cadres. Sur ces deux points, les cadres jouissent d'un avantage comparable à celui des indépendants.

Pour les limitations ressenties dans le travail (colonne III), leur situation relative est meilleure, tandis que les non-salariés voisinent avec les salariés non-cadres. On remarque également (colonne IV) la

(24) Le mini-module européen est composé de trois questions qui structurent le tableau 1. Ces questions, qui se sont stabilisées autour du milieu de la décennie 2000, résultent d'un long processus de concertation entre les instances nationales et internationales (Eurostat) chargées de suivre les questions de santé. Elles sont désormais présentes dans la plupart des enquêtes en population générale et, à ce titre, très régulièrement exploitées par les chercheurs. Le travail de documentation dont elles ont fait l'objet repose notamment sur des études de teneur médicale ou épidémiologique qui ont éclairé la portée «objectivante» ou même prédictive des déclarations de santé subjectivement perçue (DESALVo et al., 2005; ERDOGAN-CIFTCI et al., 2010; MillunPalo et al., 1997).

(25) Pour une exploitation détaillée des réponses comparées au mini module-européen voir : AlgaVa, CAVAlin, CÉlérIER (2011) et précisément le tableau 3 page 10. position des non-salariés et des cadres en matière de stress, seul critère de santé pour lequel ils se situent moins favorablement que les salariés non-cadres. Ce résultat confirme l'intérêt du chantier récemment ouvert sur le stress des indépendants (INSERM, 2011), mais il doit être lu avec réserve. La question de $H S M$ à propos du «stress » ne donne aucun contour directif à cette notion qui reste, elle-même, assez floue. Les résultats de la colonne $\mathrm{V}$ soulignent en outre la moindre propension des non-salariés (plus forte cependant que celle des cadres) à changer d'activité professionnelle pour raisons de santé. Cette dernière mesure reste toutefois générale et nous aurions besoin de précisions pour mieux comprendre l'étendue des changements qui s'offrent aux individus.

Enfin, les deux dernières colonnes du tableau (VI et VII) qui comparent le recours à deux types de soins présentent un double intérêt. Elles identifient le trait le plus distinctif de la santé (ou plutôt du comportement de santé) des non-salariés et suggèrent que leur avantage relatif diffère en nature de celui des cadres. À caractéristiques données, ces derniers ont en effet une probabilité plus faible de déclarer des maladies, des symptômes multiples ou des limitations que les autres salariés, tout en consommant plus de soins, notamment de spécialistes. Autrement dit, et comme les travaux sur les inégalités de santé l'ont régulièrement démontré, leur avantage de santé s'obtient (ou s'accompagne) de pratiques préventives et curatives plus soutenues. 
Tableau 3 : Probabilité de déclarer plusieurs caractéristiques de santé et de recourir à des soins selon le statut d'emploi

\begin{tabular}{|c|c|c|c|c|c|c|c|}
\hline & I & II & III & IV & $\mathbf{V}$ & VI & VII \\
\hline $\begin{array}{l}\text { Probabilité de } \\
\text { déclarer: }\end{array}$ & $\begin{array}{c}4 \text { symptômes } * * \\
\text { ou plus parmi } \\
9\left(\begin{array}{c}12 \text { derniers } \\
\text { mois })\end{array}\right.\end{array}$ & $\begin{array}{l}\text { Un nombre } \\
\text { de maladies } \\
\text { recodées CIM } \\
\text { supérieur ou } \\
\text { égal à } 2(\diamond) \\
\text { pour la durée } \\
\text { entière de la } \\
\text { vie }\end{array}$ & $\begin{array}{c}\text { Etre limité-e } \\
\text { dans son } \\
\text { travail pour } \\
\text { raison de santé }\end{array}$ & $\begin{array}{c}\text { Des } \\
\text { symptômes } \\
\text { de stress }(12 \\
\text { derniers mois) }\end{array}$ & $\begin{array}{l}\text { Avoir changé } \\
\text { au moins } \\
\text { une fois de } \\
\text { profession } \\
\text { dans sa vie } \\
\text { active pour } \\
\text { des raisons } \\
\text { médicales }\end{array}$ & $\begin{array}{l}\text { Avoir consulté } \\
\text { au moins } \\
\text { une fois un } \\
\text { généraliste au } \\
\text { cours des } \\
12 \text { derniers } \\
\text { mois }\end{array}$ & $\begin{array}{c}\text { Avoir consulté } \\
\text { au moins } \\
\text { une fois un } \\
\text { spécialiste au } \\
\text { cours des } \\
12 \text { derniers } \\
\text { mois }\end{array}$ \\
\hline $\begin{array}{l}\text { Non-salariés } \\
(\mathrm{n}=931)\end{array}$ & $0,65^{*}$ & $0,77^{*}$ & ns & $1,27^{*}$ & $0,60^{*}$ & $0,52 *$ & $0,84^{*}$ \\
\hline $\begin{array}{l}\text { Cadres et } \\
\text { professions } \\
\text { intellectuelles } \\
\text { supérieures } \\
(\mathrm{n}=1033)\end{array}$ & $0,70^{*}$ & $0,80^{*}$ & $0,44^{*}$ & $1,43^{*}$ & $0,46^{*}$ & ns & $1,23^{*}$ \\
\hline $\begin{array}{l}\text { Salariés } \\
\text { non-cadres } \\
(\mathrm{n}=6689)\end{array}$ & réf. & réf. & réf. & réf. & réf. & réf. & réf. \\
\hline
\end{tabular}

* Odds ratio significatif au seuil de $5 \%$.

** 4 symptômes parmi : troubles du sommeil, fatigue, troubles du comportement alimentaire, brûlures d'estomac, reflux, palpitations, tachycardie, malaises, vertiges, éblouissements, étourdissements, essoufflements, colites, douleurs intestinales, stress.

$(\diamond)$ Les nombres médian et moyen de maladies chroniques après recodage CIM (Classification internationale des maladies) parmi les 18-65 ans sont de 1 et 1,67 (respectivement 1 et 1,46 parmi les 18-65 ans actifs occupés); 41\% des 18-65 ans (et 38\% des 18-65 ans actifs occupés) présentent deux maladies recodées CIM ou plus pour toute la durée de leur vie. Le raisonnement à âge donné évite de donner à lire l'accroissement du nombre de maladies comme fruit de l'avancement en âge d'une personne (effet mécanique du temps écoulé redoublé par la probabilité croissante d'être malade avec l'âge).

Lecture : À âge, sexe, statut d'emploi, niveau de vie et couverture maladie complémentaire donnés, les non-salariés ont une probabilité inférieure de $35 \%$ à celle des salariés non-cadres de déclarer 4 symptômes ou plus (dans une liste de 9) pour les 12 mois précédant l'enquête.

Champ : Actifs occupés, 18-65 ans.

Source : HSM 2008.

Pour les non-salariés, l'avantage relatif ne découle manifestement pas de telles pratiques; soit que leurs besoins ${ }^{(26)}$ de recourir aux soins soient moindres que ceux des autres groupes, soit qu'ils les retardent davantage - avec le risque, dans ce cas, de présenter à terme un état de santé plus dégradé. Les raisons qu'ils avancent pour avoir renoncé au moins une fois à des soins au cours des douze mois écoulés sont nettement associées à l'emprise de leur travail. Tandis que cadres et autres salariés évoquent majoritairement le coût des soins (54 et $52 \%$ ), les non-salariés y font référence à parité $(37 \%)$ avec le manque de temps $(38 \%)^{(27)}$.

Le caractère moins favorable de la couverture sociale des indépendants en matière de santé peut également peser sur leur moindre consommation médicale (28). De fait, nos analyses conduites «toutes choses égales par ailleurs» montrent, en intégrant la présence ou non d'une complémentaire

(26) Du moins ceux qu'ils identifient comme tels.

(27) HSM 2008 (Drees-Insee).

(28) Le régime général reste le plus protecteur en effet (TAButeau, 2010). Le montant des compensations proposées est plus élevé, les durées de prise en charge plus longues. La réparation des accidents et maladies professionnelles n'existe pas pour les cotisants au régime social des indépendants (RSI) qui ne peuvent pas davantage bénéficier d'aménagements susceptibles de faciliter leur retour en emploi après la maladie, tel le mi-temps thérapeutique. Pour un détail des différences entre les régimes de protection se reporter à Alagava, CAVALIN, CÉLÉRIER (2011). santé dans les variables contrôlées, que le statut d'indépendant s'associe moins fréquemment à une complémentaire ${ }^{(29)}$. L'effet «statut d'emploi» n'est donc probablement pas étranger à l'effet «statut d'assuré social» confirmant que ce statut d'assuré joue un rôle crucial dans le rapport aux soins des individus; résultat qui conforte notre intérêt pour le sujet et invite à poursuivre la démonstration sur la base d'enquêtes adaptées ${ }^{(30)}$.

Pour valider l'idée d'un avantage relatif de santé des non-salariés, il reste encore à écarter l'hypothèse d'un artefact statistique qui confondrait des situations de santé très différentes, voire polarisées, parmi les composantes du groupe. On pense d'un côté aux professions libérales que les revenus et les diplômes situent à proximité des cadres salariés et, de l'autre, aux artisans, commerçants ou agriculteurs exploitants dont la santé pourrait être aussi peu favorable que celle des ouvriers, par exemple, avec lesquels ils partagent beaucoup des conditions de travail. Autrement dit, on peut penser que la bonne santé relative des non-salariés résulterait en bonne part d'inégalités sociales de santé internes au groupe, les plus favorisés en son sein (par le diplôme ou le niveau de vie) tirant vers le haut les performances moyennes de santé du groupe tout entier.

(29) Malgré la loi Madelin de 1994 qui ouvre à des déductions de charges (Perronnin et al., 2011).

(30) Par exemple et notamment, l'enquête biennale Santé et protection sociale (ESPS) gérée par l'Irdes. 
Tableau 4 : Probabilité de déclarer plusieurs caractéristiques de santé et de recourir à des soins selon le statut d'emploi décomposé

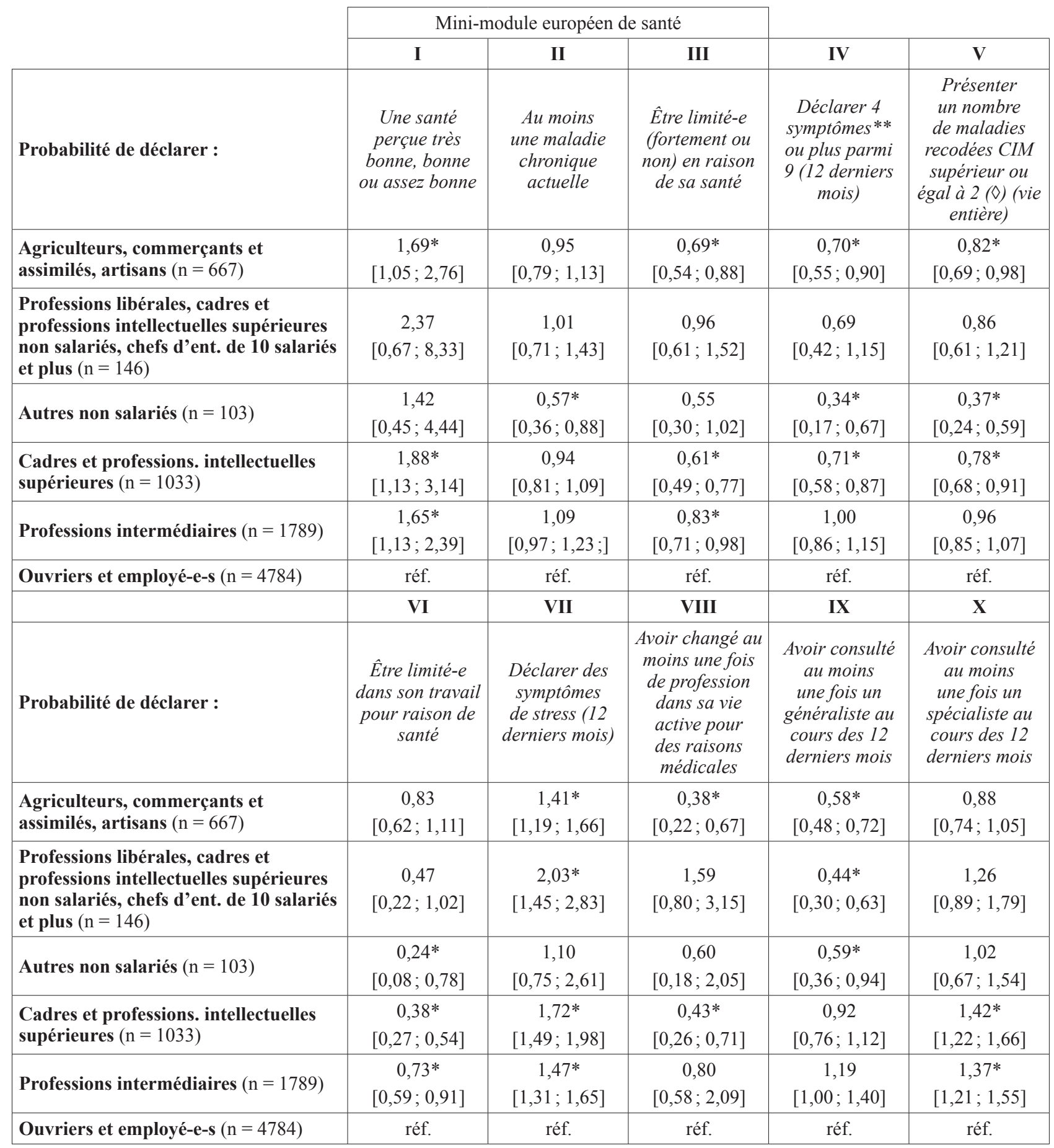

* Odds ratio significatif au seuil de $5 \%$.

** 4 symptômes parmi : troubles du sommeil, fatigue, troubles du comportement alimentaire, brûlures d'estomac, reflux, palpitations, tachycardie, malaises, vertiges, éblouissements, étourdissements, essoufflements, colites, douleurs intestinales, stress.

$(\diamond)$ Les nombres médian et moyen de maladies chroniques après recodage CIM parmi les 18-65 ans sont de 1 et 1,67 (respectivement 1 et 1,46 parmi les 18-65 ans actifs occupés); $41 \%$ des 18-65 ans (et 38\% des 18-65 ans actifs occupés) présentent deux maladies recodées CIM ou plus pour toute la durée de leur vie. Le raisonnement à âge donné évite de donner à lire l'accroissement du nombre de maladies comme fruit de l'avancement en âge d'une personne (effet mécanique du temps écoulé redoublé par la probabilité croissante d'être malade avec l'âge).

Lecture : À âge, sexe, statut d'emploi, niveau de vie et couverture maladie complémentaire donnés, être agriculteur, commerçant ou artisan plutôt qu'ouvrier ou employé multiplie par 1,69 la probabilité de déclarer être en très bonne, bonne ou assez bonne santé (1 question du mini-module européen de santé).

Champ : Actifs occupés, 18-65 ans.

Source : HSM 2008.

Nous testons la diversité interne aux non-salariés en distinguant en leur sein trois groupes : les

agriculteurs-artisans-commerçants; les professions libérales et assimilés, chefs d'entreprise de dix 
salariés ou plus; et les «autres» non-salariés ${ }^{(31)}$. Nous rapportons ces groupes à l'ensemble des salariés dans lesquels nous distinguons également trois groupes : les cadres et professions intellectuelles supérieures; les professions intermédiaires; les employé-e-s et ouvriers. Le tableau 4 livre les probabilités de déclaration de dix indicateurs de santé en prenant le sous-groupe des ouvriers et employé-e-s pour référence dans la variable explicative de statut d'emploi. Globalement, nous lisons que les non-salariés déclarent une santé relativement bonne pour tous les indicateurs des colonnes I à VIII du tableau. Les professions libérales (et assimilés) ne s'y démarquent pas et, hormis pour les déclarations de stress (colonne VII ${ }^{(32)}$ ), ne rejoignent nullement les cadres salariés. Les agriculteurs, artisans et commerçants occupent, pour ces mêmes indicateurs, une position relative plus favorable qu'attendu, proche, à de nombreux égards, des professions intermédiaires salariées.

Considérant l'ensemble des actifs de dix-huit à soixante-cinq ans, nous retrouvons les résultats que les recherches sur les inégalités sociales de santé nous laissaient anticiper. Les cadres et professions intellectuelles supérieures ressortent avec un avantage sensible. Pour la grande majorité de nos huit premiers indicateurs, ils présentent en effet de plus fortes probabilités de déclarer une santé meilleure que les autres groupes d'actifs occupés, à autres caractéristiques données. Ce résultat lisible en termes de PCS est très net et retrouve, encore une fois, le gradient connu des inégalités sociales de santé.

Mais ce gradient ne se retrouve cependant pas exactement lorsqu'intervient le statut d'emploi : à caractéristiques données et en prenant le statut d'emploi «ouvriers et employé-e-s» en référence, les cadres (salariés) et les professions libérales (non-salariés) ne jouissent pas, on l'a dit, du même privilège social en santé. Pour vérifier ce point, nous avons réalisé d'autres régressions logistiques reprenant les mêmes indicateurs de santé (variables expliquées), les mêmes variables explicatives et les mêmes sous-groupes que pour le tableau 4, mais en

(31) Ces «autres» non-salariés sont une illustration de la diversité que recouvre le statut d'indépendant : tout en ayant un statut d'indépendant au sens de la variable «Insee» de statut que nous utilisons ici, ils déclarent une catégorie socioprofessionnelle appartenant aux groupes 4 (professions intermédiaires), 5 (employés) ou 6 (ouvriers). Cet «autre» non-salariat rassemble 103 personnes dans HSM 2008 occupées à de multiples métiers comme en témoignent les catégories socioprofessionnelles recensées dans l'encadré. Citons, par exemple, les professions intermédiaires : de la santé(infimier-e-s, kinésithérapeutes), de la publicité et de la communication, de l'accueil et du gardiennage, de l'interprétariat ou encore les professions d'exécution spécialisées dans l'élevage ou le traitement forestier.

(32) Nous retrouvons ici ce que le tableau 3 indiquait déjà à propos des symptômes de stress ressentis et déclarés, à savoir le surcroît de probabilité (presque universel) de ces symptômes hors groupe des ouvriers et employés. opérant cette fois de manière séparée sur le souschamp des non-salariés d'un côté et celui des salariés de l'autre. Nous voulions comparer les inégalités de santé entre cadres et ouvriers-employés (dans le sous-champ du salariat) à celles qui distingueraient les professions libérales des agriculteurs, artisans et commerçants (dans celui de l'indépendance).

Les résultats confirment les précédents, à savoir des avantages remarquables pour les cadres ${ }^{(33)}$ mais aucune différence significative parmi les non-salariés, sur neuf des dix indicateurs considérés. Seule la probabilité d'avoir changé au moins une fois dans sa vie de profession pour raison médicale distinguerait nettement les professions libérales ${ }^{(34)}$ mais ce résultat, reposant sur des effectifs trop réduits, ne présente pas une robustesse statistique suffisante pour qu'on puisse s'y fier sans réserve. Quoi qu'il en soit, nous constatons clairement que la variable PCS n'est plus universellement opérante dès que le statut d'emploi est pris en compte. C'est là un résultat robuste de nos analyses.

\section{Quand la maladie survient}

Le dernier mécanisme susceptible de jouer dans la santé relativement meilleure des indépendants concerne la sélection à l'entrée ou en sortie du statut. Ceux qui présenteraient une santé précaire ou se sentiraient le moins assurés de ce point de vue s'écarteraient d'un statut qui les expose davantage et en sortiraient aux premiers doutes. On retrouverait donc, sur ce statut, les travailleurs dont la santé est la moins mauvaise expliquant leur position globalement favorable malgré un âge moyen relativement élevé. Nos explorations sur ce point sont fortement limitées par l'absence de données longitudinales. Pour approcher cette question cependant, nous avons tiré partie des données rétrospectives de l'enquête SIP (2006) qui sollicite la mémoire des enquêtés pour reconstituer leurs itinéraires professionnels et recenser les problèmes de santé qu'ils disent avoir rencontrés tout au long de leur vie. Avec la prudence qu'impose une telle matière, nous avons cherché les effets comparés de la maladie selon le statut.

En effet, si les indépendants jouissent d'un état de santé relativement favorable malgré leur âge, ils ne sont pourtant pas épargnés par la maladie, qui les touche comme les autres groupes. Pour n'en donner qu'un exemple - lié d'ailleurs à leur âge moyen -, ils sont concernés par 9 des 60 cancers

(33) Odds ratios pour les cadres et professions intellectuelles supérieures (référence : ouvriers et employés), en respectant l'ordre des colonnes I à X du tableau $4: 1,79 ; \mathrm{ns} ; 0,62 ; 0,73$; 0,$80 ; 0,39 ; 1,78 ; 0,42 ;$ ns $; 1,43$.

(34) Odds ratio : 4,96 (le statut de référence étant alors, dans le sous-champ des non-salariés, celui des agriculteurs, artisans et commerçants). 
recensés dans l'enquête $H S M$ en 2008, alors que les cadres dont les effectifs sont équivalents le sont pour 4 d'entre eux. Cette seconde partie de notre article explore donc ce qu'il advient quand - salarié ou non-salarié - la maladie, le handicap ou les limitations de diverses natures viennent troubler le cours de la vie professionnelle. Nous avons suivi les effets de ces problèmes sur l'itinéraire professionnel en sélectionnant trois effets a priori possibles pour lesquels les données de l'enquête fournissaient les informations les plus adaptées. Nous traitons le premier sous le registre de l'inflexion de la carrière, que la littérature a abordée dans les termes d'une précarisation des situations d'emploi. Les deux effets suivants, l'interruption momentanée de l'activité professionnelle ou la modulation de l'organisation temporelle du travail, sont regroupés et analysés comme aménagements des activités professionnelles.

\section{Les effets sur l'itinéraire professionnel}

L'impact de telle ou telle maladie sur l'itinéraire professionnel est une question que se posent tous les spécialistes de ladite maladie dans leur suivi du devenir des malades. La remarque vaut, même si cette préoccupation reste marginale rapportée aux suivis des effets des traitements, en particulier si les risques létaux de la maladie sont élevés. Les cancers en donnent un exemple archétypal (CÉLÉRIER, 2008). La veine anglo-saxonne interroge particulièrement les effets des maladies chroniques en termes de retour à l'emploi, plus ou moins réalisé et plus ou moins «retardé» (SPELTEN et al., 2002). En France, la réflexion est plus large et est marquée par le constat réalisé depuis les années 1980 de fortes différences de santé entre les chômeurs et les actifs, les premiers présentant toujours un état de santé moins favorable que les seconds (Khlat, SERmet, 2004).

La recherche des causalités croisées qui expliquent ce constat a installé une tradition d'analyse des impacts de la maladie tant vers le chômage que vers l'inactivité en détaillant le plus souvent les situations des femmes et des hommes (MesRINe, 2000 ; SAURELCubizolles, 2001; Malenfant et al., 2004; Jusot et $a l ., 2006)$. Ces études concluent généralement à un coût pour les malades, même si les estimations en sont très variables d'une étude à l'autre et qu'elles peuvent aussi se lire de façon encourageante. Une étude récente sur l'impact du cancer montre ainsi que beaucoup de personnes y survivent aujourd'hui et que, dans leur grande majorité, elles retrouvent deux ans après le diagnostic des conditions de vie proches de celles de la population générale (EicHENBAumVoline et al., 2008, pp. 126-127) (35).

(35) Reste néanmoins le désavantage des travailleurs manuels toujours plus sévèrement sanctionnés par la maladie y compris quand le pronostic est favorable.
Reprenant les pistes ouvertes par les travaux français, nous examinons trois types d'inflexion de l'itinéraire professionnel : le chômage, l'inactivité ainsi que l'emploi instable (36) auxquels nous ajoutons un éventuel changement de statut des nonsalariés vers le salariat et réciproquement. Nous partons des maladies telles qu'elles sont déclarées par les enquêtés de $S I P$, en distinguant les déclarants selon qu'ils occupaient un emploi non-salarié ou salarié l'année $\mathrm{n}-1$, pour une maladie ayant commencé l'année $n$. Nous enregistrons leurs positions respectives à l'issue de cette année $(\mathrm{n})$, un an $(n+1)$ puis deux ans $(n+2)$ après la survenue de la maladie. Le tableau 5 présente les résultats en distinguant à gauche le devenir des indépendants et à droite celui des salariés. Le tableau distingue également les personnes selon qu'elles ont atteint ou non l'âge de cinquante ans, car le passage à l'inactivité est plus fréquent lorsqu'une maladie survient à un âge plus avancé.

Tableau 5 : Situation professionnelle l'année de la maladie, un an après et deux ans après, en fonction de l'âge et du statut d'emploi un an avant la maladie

\begin{tabular}{|l|c|c|c|}
\hline $\begin{array}{c}\text { Non } \\
\text { salariés en } \\
\mathbf{n - 1 , 5 0} \text { ans } \\
\text { ou plus } \\
(\mathbf{n}=\mathbf{4 3 4})\end{array}$ & $\mathrm{n}$ & $\mathrm{n}+1$ & $\mathrm{n}+2$ \\
\hline & $\%$ & $\%$ & $\%$ \\
\hline Chômage & 1 & 1 & 0 \\
\hline Inactif & 24 & 36 & 46 \\
\hline Non salarié & 73 & 61 & 52 \\
\hline Salarié & 0 & 0 & 0 \\
\hline $\begin{array}{l}\text { Emplois } \\
\text { courts }\end{array}$ & 1 & 1 & 1 \\
\hline
\end{tabular}

\begin{tabular}{|l|c|c|c|}
\hline $\begin{array}{c}\text { Salariés en } \\
\text { n-1, 50 ans } \\
\text { ou plus (n } \\
=\mathbf{1 ~ 8 1 7 )}\end{array}$ & $\mathrm{n}$ & $\mathrm{n}+1$ & $\mathrm{n}+2$ \\
\hline & $\%$ & $\%$ & $\%$ \\
\hline Chômage & 1 & 2 & 4 \\
\hline Inactif & 22 & 36 & 48 \\
\hline Non salarié & 0 & 0 & 0 \\
\hline Salarié & 76 & 61 & 48 \\
\hline $\begin{array}{l}\text { Emplois } \\
\text { courts }\end{array}$ & 0 & 1 & 0 \\
\hline
\end{tabular}

\begin{tabular}{|l|c|c|c|}
\hline $\begin{array}{c}\text { Non } \\
\text { salariés en } \\
\text { n-1, moins } \\
\text { de 50 ans } \\
(\mathbf{n}=\mathbf{5 5 4})\end{array}$ & $\mathrm{n}$ & $\mathrm{n}+1$ & $\mathrm{n}+2$ \\
\hline & $\%$ & $\%$ & $\%$ \\
\hline Chômage & 1 & 1 & 0 \\
\hline Inactif & 8 & 13 & 15 \\
\hline Non salarié & 90 & 82 & 77 \\
\hline Salarié & 0 & 1 & 4 \\
\hline $\begin{array}{l}\text { Emplois } \\
\text { courts }\end{array}$ & 1 & 2 & 4 \\
\hline
\end{tabular}

\begin{tabular}{|l|c|c|c|}
\hline $\begin{array}{c}\text { Salariés en } \\
\text { n-1, moins } \\
\text { de 50 ans } \\
(\mathbf{n = 4 6 7 6 )}\end{array}$ & $\mathrm{n}$ & $\mathrm{n}+1$ & $\mathrm{n}+2$ \\
\hline & $\%$ & $\%$ & $\%$ \\
\hline Chômage & 1 & 2 & 2 \\
\hline Inactif & 8 & 13 & 18 \\
\hline Non salarié & 0 & 0 & 0 \\
\hline Salarié & 89 & 83 & 77 \\
\hline $\begin{array}{l}\text { Emplois } \\
\text { courts }\end{array}$ & 1 & 2 & 3 \\
\hline
\end{tabular}

Lecture : Parmi l'ensemble des maladies déclarées par les personnes qui étaient non-salariées en emploi long et âgées de 50 ans ou plus l'année $\mathrm{n}-1,46 \%$ sont inactives en $\mathrm{n}+2$.

Champ : Ensemble des maladies ayant affecté des personnes qui se trouvaient en emploi long, salarié ou non-salarié l'année précédente $(\mathrm{n}=7481)$.

Source : SIP 2006 (Drees-Dares).

(36) Pour l'enquête SIP, l'emploi long se définit comme un même emploi tenu cinq ans et plus, l'emploi court comme un emploi tenu moins de cinq ans, avec éventuellement du chômage et de l'inactivité (de moins d'un an), le chômage correspondant lui-même à une période d'un an minimum et l'inactivité comme sortie de l'emploi pendant au moins un an. 
Au total, quelque 7500 maladies sont déclarées ${ }^{(37)}$ dans SIP qui concernent très majoritairement les salariés et, plus précisément, les salariés de moins de cinquante ans qui sont, et de loin, les plus représentés dans l'enquête. Les données du tableau 5 font ressortir deux résultats marquants. D'une part, la maladie n'engage aucun mouvement vers l'indépendance de la part des salariés, et ce, quel que soit leur âge (38). Si mouvement il y a, il va du non-salariat vers le salariat, du moins pour les plus jeunes des non salariés. Si l'on additionne les emplois courts et les emplois longs salariés, $8 \%$ des plus jeunes non-salariés ont changé de statut deux ans après l'événement de santé. Cette observation va plutôt dans le sens d'un effet travailleur sain, mais ne peut cependant en décider complètement.

D'abord, il ne touche qu'un aspect de la question en ignorant, par définition, les salariés en bonne santé devenus indépendants. Ensuite, observé deux ans après la maladie, il peut résulter d'autres causes, notamment conjoncturelles ${ }^{(39)}$; l'effet peut donc s'être dilué. Le second résultat du tableau 5 vient de la confrontation des parties supérieures et inférieures du tableau - de part et d'autre de cinquante ans - d'où ressort le caractère structurant de l'âge, supérieur à celui du statut. Les devenirs des salariés et des non-salariés de cinquante ans et plus sont comparables et logiquement orientés vers l'inactivité qui prend sans doute ici la forme de la retraite. Même similarité pour les plus jeunes, bien que les salariés qui aient été malades se retrouvent plus fréquemment au chômage que leurs équivalents non-salariés.

Sur le chapitre des inflexions de la carrière, on peut encore apprécier le risque de sortie d'emploi à la suite d'une maladie en contrôlant de l'âge de survenue de la maladie, du sexe, du type de la maladie et du statut dans l'emploi l'année précédant la maladie. Détaillé dans le tableau 6, ce risque n'est pas plus significativement différent pour les non-salariés que pour les salariés en emploi long, l'année même de la survenue de la maladie, tout comme l'année suivante. Il ne devient sensible que deux ans après, lorsque les non-salariés voient leur probabilité de sortir de l'emploi réduite d'un tiers par rapport à un salarié en emploi long et à autres caractéristiques données. L'effet le plus net dont témoigne le tableau 6 concerne cependant l'instabilité de l'emploi qui aggrave lourdement le risque de perte d'emploi, bien plus que le statut

(37) Nous ne considérons pas le devenir des personnes qui se trouvaient en emplois courts à $\mathrm{n}$ - 1 (1 642 maladies déclarées). Les fréquences qui apparaissent aux points $n, n+1$ et $n+2$ de la ligne du tableau 5 «Emplois courts» ne traduisent ainsi que les arrivées des non-salariés et des salariés sur ce type d'emploi. (38) Les enquêtes qualitatives présentent parfois le passage vers le travail indépendant comme une solution mise en œuvre par les personnes malades. Pour les personnes ayant déclaré un cancer, voir CHASSAING et al. (2011).

(39) Sans parler bien sûr des aléas de la mémoire. proprement dit. Rencontrer la maladie quand on travaille dans des emplois instables multiplie par près de quatre les risques de sortie de l'emploi.

Tableau 6 : Sorties de l'emploi dans les années qui suivent le début d'une maladie

\begin{tabular}{|c|c|c|c|c|c|c|}
\hline \multirow{3}{*}{$\begin{array}{l}\text { Situation un } \\
\text { an avant le } \\
\text { problème de } \\
\text { santé déclaré }\end{array}$} & \multicolumn{6}{|c|}{$\begin{array}{l}\text { Proportion de personnes qui ne sont plus } \\
\text { en emploi }\end{array}$} \\
\hline & \multicolumn{2}{|c|}{$\begin{array}{l}\text { L'année de } \\
\text { début du } \\
\text { problème de } \\
\text { santé }\end{array}$} & \multicolumn{2}{|c|}{$\begin{array}{l}\text { L'année } \\
\text { suivante }\end{array}$} & \multicolumn{2}{|c|}{$\begin{array}{l}\text { Deux ans } \\
\text { après }\end{array}$} \\
\hline & En \% & & En \% & & & \\
\hline $\begin{array}{ll}\text { Empl } \\
\text { non-s }\end{array}$ & 16,2 & $\mathrm{~ns}^{* *}$ & 24,8 & ns** & 29,0 & 0,65 \\
\hline $\begin{array}{l}\text { Emploi long } \\
\text { salarié }\end{array}$ & 13,1 & 1 & 21,4 & 1 & 28,8 & 1 \\
\hline $\begin{array}{l}\text { Emploi court } \\
\text { salarié }\end{array}$ & 30,3 & 4,2 & 40,9 & 4,1 & 46,0 & 3,7 \\
\hline
\end{tabular}

* OR : Odds ratios (significatifs au seuil de $5 \%$ ) issus de régressions logistiques déterminant la probabilité de sortir de l'emploi en fonction de l'âge de survenue de la maladie, du sexe, du type de la maladie et du statut dans l'emploi l'année précédant la maladie.

** ns : OR non significatif au seuil de $5 \%$.

Lecture : Pour une personne non-salariée en emploi long en $\mathrm{n}-1$, la probabilité de ne plus être en emploi en $n+2$ pour une maladie survenue l'année n est de $35 \%$ inférieure à ce qu'elle est pour un salarié en emploi long.

Champ : Ensemble des problèmes de santé ayant affecté des personnes qui se trouvaient en emploi long salarié, non-salarié ou en emploi court l'année précédente $(\mathrm{n}=9123)$.

Source : SIP 2006 (Drees-Dares).

\section{Aménager l'activité professionnelle}

Dans ce second type d'impact de la maladie, on observe non plus des ruptures ou des inflexions de l'itinéraire, mais des modulations fortement encadrées par les systèmes assurantiels (interruptions ou non de l'activité pour ce qui concerne l'arrêt temporaire de travail) et relatives à la nature de l'activité (aménagement des dimensions temporelles dont nous avons rappelé les spécificités partagées dans la première partie).

\section{Interrompre son activité}

Du point de vue des arrêts temporaires de travail, la comparaison des salariés et des non-salariés révèle des comportements radicalement différents qui font écho à ce qui s'observait plus haut en matière de consommation de soins. La probabilité des non-salariés de s'arrêter à l'occasion d'une maladie ${ }^{(40)}$ est de deux tiers inférieure à celle des salariés en emploi long, que l'arrêt soit unique ou réitéré (voir tableau 7). Les non-salariés semblent donc maintenir leur activité professionnelle bien plus que ne le font les salariés, mais leurs droits respectifs en la matière sont si différents que la

(40) Il ne s'agit ici que des maladies que les enquêtés jugent comme étant les plus marquantes dans leur vie, selon le mode d'interrogation de l'enquête $S I P$, soit $83 \%$ de l'ensemble des maladies enregistrées. 
comparabilité de leurs comportements n'est plus complètement assurée.

Tableau 7 : Conséquences de la maladie en termes d'arrêt de travail et de changement dans l'itinéraire professionnel

\begin{tabular}{|l|c|c|c|c|}
\cline { 2 - 5 } \multicolumn{1}{c|}{} & \multicolumn{3}{c|}{ La maladie a occasionné... } \\
\hline \multirow{2}{*}{ Situation un an avant } & \multicolumn{2}{|c|}{$\begin{array}{c}\text {...un ou } \\
\text { plusieurs arrêts } \\
\text { de travail * }\end{array}$} & $\begin{array}{c}\text { 年es } \\
\text { conséquences } \\
\text { sur la situation } \\
\text { professionnelle* }\end{array}$ \\
\cline { 2 - 5 } & En \% & OR1** & En \% & OR2*** \\
\hline $\begin{array}{l}\text { Emploi long } \\
\text { non-salarié }\end{array}$ & 29,4 & 0,33 & 35,8 & ns \\
\hline Emploi long salarié & 52,9 & 1 & 34,0 & 1 \\
\hline Emploi court & 47,2 & 0,66 & 41,9 & 1,4 \\
\hline
\end{tabular}

* Exploitation de deux questions de l'enquête SIP posées à propos de trois maladies au plus, laissées au choix de l'enquêté parmi celles qui lui «semblent le plus liées à (sa) vie professionnelle, qu'il s'agisse de causes ou de conséquences».

** OR1 : Odds ratio issu d'une régression logistique portant sur la probabilité de prendre un ou plusieurs arrêts de travail liés à la maladie décrite en fonction de l'âge de survenue de la maladie, du sexe, du type de la maladie et du statut dans l'emploi.

*** OR2 : Même chose, mais à propos de la probabilité de déclarer que la maladie a entraîné des conséquences sur la situation professionnelle.

Lecture : Pour une personne non-salariée en emploi long l'année précédant la maladie, la probabilité de prendre un ou plusieurs arrêts de travail en lien avec la maladie décrite est inférieure de deux tiers à celle qui caractérise un salarié en emploi long.

Champ : Ensemble des maladies ayant affecté des personnes qui se trouvaient en emploi long (salarié ou non-salarié) ou en emploi court l'année précédant la maladie.

Source : SIP 2006 (Drees-Dares).

Au même titre que la consommation de soins, l'arrêt de travail est fortement dépendant des dispositions légales. Pour les salariés, cette interruption temporaire est depuis longtemps organisée par le droit ${ }^{(41)}$ qui suspend leur contrat de travail et complète la perte momentanée de leurs revenus par des indemnités journalières financées par les salariés en activité et les employeurs. La situation des non-salariés est bien différente et reste moins avantageuse, nous l'avons dit, et ce malgré les restrictions récemment imposées au régime général ${ }^{(42)}$. Seuls les artisans et les commerçants sont assujettis et bénéficient d'une protection contre le risque d'interruption de l'activité et perçoivent des indemnités journalières - quoique moins favorables en durée de perception et en valeur que celles délivrées aux salariés. Les professions libérales s'assurent individuellement, mais sans obligation et dans des conditions variables du point

(41) Sylvie Bourgeot et Michel Blatman (2009, p. 288) font remonter cette règle à une jurisprudence de 1934 (Ch. civ., 3 décembre, Hôtel Terminus, PLM c/dame Spagnoli).

(42) Le décret du 26 décembre 2011 instaure par exemple un mode de calcul des indemnités journalières moins favorable à l'assuré (JORF n 0299, 27 décembre 2011, p. 22 309). Il fait suite à deux décrets du 29 octobre 2010 qui avaient déjà révisé à la baisse le calcul des prestations en espèces. Citons encore l'article 105 de la loi de finances pour $2012\left(\mathrm{n}^{\circ} 2011-1977 \mathrm{du}\right.$ 28 décembre 2011) qui instaure un jour de carence en cas de maladie dans la fonction publique. de vue de la couverture proposée et des niveaux de cotisation. La situation est identique pour les exploitants agricoles ${ }^{(43)}$, hormis pour les maladies ou les accidents dont l'origine professionnelle est reconnue et pour lesquels ils perçoivent des indemnités journalières.

Par ailleurs, les risques qui s'attachent à la suspension temporaire de l'activité des salariés et des non-salariés ont peu à voir les uns avec les autres. Les salariés sont principalement menacés par le licenciement et, si le risque n'est pas nul, la loi du 12 juillet $1990^{(44)}$ qui affirme que la maladie ne peut à elle seule être une cause de rupture du contrat de travail, leur assure une plus grande sécurité de ce point de vue. Pour les indépendants, les risques sont tout autres. La perte de revenus n'est pas compensée, nous l'avons vu, et l'impossibilité de tenir l'activité constitue une menace, assurément, en fragilisant le réseau de relations (dont les clients) dans lequel elle s'insère. Il est probable que l'arrêt du travail ne constitue qu'une solution de dernière instance pour les indépendants.

La possibilité de suspendre momentanément l'activité professionnelle est donc sensible au statut dans la mesure où les droits qui lui sont associés la rendent plus ou moins effective et plus ou moins dangereuse pour la suite de la carrière. L'arrêt de travail des non-salariés et celui des salariés y prennent des acceptions très différentes et difficilement comparables. À qui établirait un lien ferme, voire vertueux, entre le maintien dans l'emploi et une plus faible (et donc économiquement incitative) protection sociale, on pourrait opposer le coût que ce maintien suppose, pour l'individu et pour la collectivité, en termes de retard dans les soins et finalement d'aggravation possible de l'état de santé. On retrouve ici la question soulevée plus haut en constatant que les non-salariés sous-consomment sensiblement des consultations de spécialistes et (surtout) de généralistes sur une période de douze mois écoulés. L'enquête SIP ne permet pas pour le moment de renseigner ces aspects qui doivent cependant rester présents dans l'interprétation des résultats.

\section{Aménager le temps de travail}

Le second aménagement considéré concerne le temps de travail dont nous avons vu les spécificités pour les indépendants, que nous avons traduites en termes «d'emprise». Nous avons voulu savoir comment de telles spécificités se traduisaient dans le contexte de la maladie, savoir donc si elles constituaient des contraintes supplémentaires en raison du nombre d'heures et de l'emprise du

(43) Des négociations sont en cours pour mettre en place des indemnités journalières concernant les maladies autres que celles reconnues d'origine professionnelle.

(44) Devenu l'article L. 1132-1 du Code du travail. 
travail, ou si les non-salariés y trouvaient des ressources. La maladie et les traitements qu'elle impose sont de nature en effet à susciter de vives tensions dans l'organisation temporelle de l'activité indépendante et nous souhaitions donc identifier les ajustements qu'elle impose. Pour mener l'analyse, nous distinguons, parmi les 879 non-salariés interrogés dans $S I P$, les 211 personnes qui déclarent au moins une maladie chronique en procédant de même pour les cadres et pour les autres salariés. Le tableau 8 en présente les résultats sur quelques dimensions-clefs du temps de travail en dissociant, pour chaque groupe considéré, les déclarations des personnes ayant rapporté avoir une maladie chronique (colonne MC) de celles des autres. Les effectifs limités invitent à une grande prudence dans l'interprétation. En dépit de ces réserves, nous présentons ces résultats pour les quelques différences intéressantes qu'ils signalent, que nous prenons comme autant de pistes à reprendre par des études à venir pour confirmation ou reformulation. Le tableau 8 sert donc ici la formation d'hypothèses très provisoires.

Tableau 8 : Maladies chroniques des actifs en emploi et conditions de travail

\begin{tabular}{|l|c|c|c|c|c|c|}
\cline { 2 - 6 } \multicolumn{1}{l|}{} & Non salariés & \multicolumn{2}{|c|}{ Cadres } & \multicolumn{2}{c|}{$\begin{array}{c}\text { Autres } \\
\text { salariés }\end{array}$} \\
\cline { 2 - 7 } & \multicolumn{5}{|c|}{ Maladie chronique } \\
\cline { 2 - 7 } & Oui & Non & Oui & Non & Oui & Non \\
\hline Effectifs & 211 & 668 & 252 & 762 & 1681 & 4539 \\
\hline $\begin{array}{l}\text { Nombre d'heures de } \\
\text { travail au cours de } \\
\text { la dernière semaine } \\
\text { travaillée }\end{array}$ & 47,9 & 49,4 & 41,0 & 41,6 & 35,1 & 36,3 \\
\hline $\begin{array}{l}\text { Travail la nuit (toujours } \\
\text { ou souvent) }\end{array}$ & $4 \%$ & $8 \%$ & $2 \%$ & $3 \%$ & $9 \%$ & $9 \%$ \\
\hline $\begin{array}{l}\text { Travail hebdomadaire } \\
\text { d'une durée supérieure } \\
\text { à 48 heures (toujours ou } \\
\text { souvent) }\end{array}$ & $60 \%$ & $58 \%$ & $29 \%$ & $29 \%$ & $9 \%$ & $8 \%$ \\
\hline $\begin{array}{l}\text { Horaires irréguliers, } \\
\text { difficilement prévisibles } \\
\text { (toujours ou souvent) }\end{array}$ & $28 \%$ & $31 \%$ & $16 \%$ & $18 \%$ & $10 \%$ & $12 \%$ \\
\hline $\begin{array}{l}\text { Travail physiquement } \\
\text { exigeant }\end{array}$ & $52 \%$ & $49 \%$ & $17 \%$ & $14 \%$ & $40 \%$ & $32 \%$ \\
\hline Travail sous pression & $29 \%$ & $30 \%$ & $53 \%$ & $43 \%$ & $29 \%$ & $23 \%$ \\
\hline
\end{tabular}

Lecture : Les non-salariés déclarant au moins une maladie chronique travaillent en moyenne 47,9 heures par semaine. Ceux qui ne déclarent aucune pathologie travaillent 49,4 heures. $52 \%$ des premiers estiment que leur travail est physiquement exigeant contre $49 \%$ des seconds. Champ : Actifs occupés de 15 ans et plus.

Source : SIP 2006 (Drees-Dares).

Les données de ce tableau 8 confirment déjà nettement l'impact de la maladie quel que soit le statut d'emploi considéré. Les malades jugent toujours plus que les autres personnes non-malades et de même statut que leur travail est «physiquement exigeant» (ligne 5). Ce résultat est généralement compris comme la résultante de deux mécanismes : les problèmes de santé rendent plus sensibles à ce type de pénibilités et ces dernières contribuent à dégrader la santé(45). Notons toutefois que les non-salariés signalent beaucoup plus souvent se heurter à ce type d'exigences physiques que les autres catégories d'actifs en emploi (qu'ils aient ou non déclaré une maladie chronique).

Qu'ils soient malades ou non, les non-salariés déclarent toujours les durées hebdomadaires de travail les plus élevées (lignes 1 et 3 ). Ils pratiquent par ailleurs moins souvent des horaires irréguliers lorsqu'ils sont malades (plutôt que non-malades), mais c'est aussi le cas des cadres et des autres salariés. Pour le travail de nuit (ligne 2) en revanche, l'écart entre non-malades et malades parmi les non-salariés paraît plus sensible que pour les autres statuts d'emploi. Malades, les non-salariés sont deux fois moins nombreux que les non-malades à travailler toujours ou souvent la nuit. Contrôlés par l'âge, le sexe et le secteur d'activité, ces résultats ne sont toutefois pas significatifs. Le statut «n'explique pas» les différences d'exposition au travail de nuit des non-salariés malades par rapport à ceux qui ne le sont pas.

Il nous semble utile cependant de conserver ces résultats dans le cadre de la démarche exploratoire qui est ici la nôtre et que nous avons rappelée plus haut, dont l'objectif est de former des hypothèses provisoires susceptibles d'orienter les recherches à venir. De ce point de vue même limité, les données du tableau 8 suggèrent que le maintien d'un nombre élevé d'heures de travail de la part des non-salariés malades pourrait s'accompagner d'un ajustement de leurs expositions : aux horaires atypiques (dans des proportions équivalentes à celles des salariés) et au travail de nuit. Nous aurions ici un effet patent de l'autonomie d'organisation du travail que les indépendants présentent classiquement comme une caractéristique de leur activité et que l'on retient parfois comme différence majeure relativement aux salariés. Or, dans un raisonnement toutes choses égales par ailleurs, la variable statut n'est pas significativement corrélée à des ajustements du temps de travail en cas de maladie. Autrement dit, les suites de la maladie se révèlent une mise à l'épreuve intéressante de cette autonomie supposée. En examinant les différentes formes d'ajustements du temps de travail, nous pourrions en apprécier la réalité et l'ampleur ou, au contraire, la contester.

Les non-salariés semblent moins exposés au «travail sous pression» que les cadres (ligne 6, tableau 8), caractéristique généralement corrélée

(45) De là, on comprend le choix des enquêtes statistiques françaises sur les conditions de travail de valoriser l'appréciation subjective des personnes à propos de conditions objectivables de travail. Les Suédois, par exemple, ont pris un tout autre parti en décrivant les conditions de travail à partir de seules mesures objectives : poids soulevés, température, décibels, etc. : voir Gollac, Volkoff (2010). Et sur le phénomène d'endogénéité des déclarations sur les conditions de travail, voir COUTROT, WolfF (2005). 
aux risques psychosociaux. Par ailleurs, les cadres et autres salariés, lorsqu'ils sont malades, déclarent sensiblement plus souvent «travailler sous pression » que lorsqu'ils sont en bonne santé (53\% vs $43 \%$ et $29 \%$ vs $23 \%$ respectivement de ces deux groupes de salariés déclarent travailler sous pression lorsqu'ils sont malades vs non-malades). En revanche, les non-salariés malades déclarent aussi souvent que leurs homologues non-malades travailler sous pression. A minima, on peut en conclure que malgré la maladie, le sentiment de travailler sous pression ne gagne pas de terrain parmi les non-salariés, alors que pour tous les autres salariés cette dégradation est sensible. Ce peut être le signe qu'avec la maladie, des possibilités d'organisation et d'aménagement du travail - moins accessibles aux salariés en pareil cas - sont mobilisées par les non-salariés pour moduler la pression ressentie au travail.

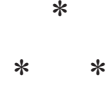

Nos analyses confirment que les indépendants déclarent une meilleure santé que les salariés et précisent cet avantage relatif. Les indépendants déclarent une meilleure santé que les salariés noncadres, mais globalement moindre que les salariés cadres qui conservent leurs prérogatives en la matière. Dans cette position intermédiaire, les indépendants se trouvent également souvent à proximité des déclarations des professions intermédiaires salariées. Pour certaines variables de santé, cet avantage relatif est largement partagé entre les sous-groupes qui composent le statut d'indépendant. Il en va ainsi des déclarations de symptômes et du recours aux généralistes toujours moins fréquent que pour les salariés, quelle que soit la PCS de ces derniers. Pour les autres variables, les différences associées au statut sont moins tranchées et le jeu classique de la PCS sur l'état de santé reprend ses droits, les cadres retrouvant leur position plus favorable.

L'avantage de santé des indépendants, s'il est bien avéré, ne se présente cependant pas de façon parfaitement homogène pour tous les sous-groupes qui composent le statut. Mais, point intéressant de nos analyses, les différences observées ne retrouvent pas le gradient classique de santé des salariés. Précisément, les professions libérales ne jouissent pas des avantages qu'une dotation supérieure en diplômes et en revenus laissait supposer par rapport aux agriculteurs, artisans et commerçants. Du point de vue de la santé, ils ne sont pas les «cadres» des indépendants. Même s'ils se disent plus souvent que tous les autres groupes en bonne santé, le nombre de leurs maladies ou leurs déclarations de stress invitent à nuancer le tableau. Seul le recours aux spécialistes les distingue positivement des autres sous-groupes indépendants.

Ainsi, la prise en compte du statut transforme ou déplace les résultats obtenus à partir des PCS qui ne sont plus si universellement opérantes. Disant cela, nous ne postulons pas une causalité directe et univoque entre le statut et la santé. Nous suggérons plutôt que le statut, précisément celui de non-salarié, abrite des dimensions dont les effets sont patents sur les déclarations de santé et les comportements de soin. Ainsi en va-t-il du moindre recours aux généralistes ou aux arrêts maladie, ces deux exemples montrant combien les alternatives qui s'ouvrent au travailleur changent selon la façon dont ses droits sont constitués et, donc - dans un pays comme la France structuré par des principes de type bismarckien ${ }^{(46)}$ - selon son statut d'emploi. On peut enfin se demander si la façon même de déclarer sa santé n'en est pas affectée en pensant à la forte probabilité de déclarer des symptômes de stress, des perceptions de l'état de santé plutôt favorables et une satisfaction au travail notablement plus élevée que les autres groupes professionnels. Plusieurs travaux récents ont enregistré de telles spécificités de déclarations sur d'autres aspects de l'activité indépendante (RIESCO, 2012; BURRI, 2011; Bessière, Gollac, 2007; Gollac, 2005; Célérier, 2002). La santé pourrait être l'occasion d'une autre singularité dont la compréhension contribuerait utilement à la réflexion plus large sur la façon dont la santé se vit et se dit.
(46) La Belgique, les Pays-Bas, l'Allemagne et l'Autriche structurent également leurs droits de santé sur ces mêmes principes traditionnellement distingués des principes «beveridgiens» reposant sur une base universelle plutôt que professionnelle et un financement à partir de l'impôt. Les pays scandinaves, l'Irlande et le Royaume-Uni mettent en œuvre cette seconde option. 


\section{Bibliographie}

Algava E., Cavalin C., Célérier S. (2011), «La santé des indépendants : un avantage relatif à interpréter», Document de travail, $\mathrm{n}^{\circ} 150$, Noisy-le-Grand, Centre d'études de l'emploi.

Algava E., Vinck L. (2009), «Les conditions de travail des non-salariés en $2005 »$, Premières Synthèses, n ${ }^{\circ} 50.1$.

Amossé T., Daubas-Letourneux V., Le Roy F., Meslin K., Barragan K. (2012), «Les accidents du travail et problèmes de santé liés au travail dans l'enquête SIP. (In)visibilités et inscriptions dans les trajectoires professionnelles », Rapport de recherche, $\mathrm{n}^{\circ} 76$, Noisy-le Grand, Centre d'études de l'emploi.

Bessière C., De Paoli C., Gouraud B., Roger M. (2011), «Les agriculteurs et leur patrimoine : des indépendants comme les autres?», Économie et statistique, ${ }^{\circ} 444-445$, pp. 55-74.

Bessière C., Gollac S. (2007), «Le silence des pratiques. La question des rapports de genre dans les familles d'“indépendants"», Sociétés \& représentations, vol. 24, $\mathrm{n}^{\circ} 2$, pp. 43-58.

BEH (2011), «Inégalités sociales de santé», Bulletin d'épidémiologie hebdomadaire, $\mathrm{n}^{\circ}$ 8-9, pp. 73-100.

Bourgeot S., Blatman M., (2009), L'état de santé du salarié, Paris, Éditions Liaisons.

Burri S. (2011), «La conciliation de la vie professionnelle, privée et familiale», in Milewski F., Périvier H., Les discriminations entre les femmes et les hommes, Paris, Presses de Sciences Po, pp. 253-280.

Cambois E., Laborde C., Robine J.-M. (2008), «La "double peine" des ouvriers : plus d'années d'incapacité au sein d'une vie plus courte», Population et sociétés, $\mathrm{n}^{\circ} 441$.

CAvalin C., Célérier S. (2012a), «Une mesure de la santé à l'âge du travail. Approche du travail par la santé à partir de l'enquête Événements de vie et santé (EVS, Drees, 2005-2006)», Rapport de recherche, $\mathrm{n}^{\circ}$ 78, Noisy-leGrand, Centre d'études de l'emploi.

Cavalin C., Célérier S. (2012b), «Mesurer les dimensions mentales de la santé au travail. Sous quels angles?», in Stroobants M. (ed.), Mesure et démesures du travail, Bruxelles, l'Université Libre de Bruxelles, pp. 185-195.

CÉLÉRIER S. (2008), «Cancer et activités professionnelles : les enseignements de 30 ans de littérature internationale sur le thème», Santé et sociologie, n 38 , pp. 201-216.

CÉLÉRIER S. (2002), “"Micro-entreprises" : entre contraintes et expression de soi», in Insee Méthode, $\mathrm{n}^{\circ} 99$, pp. 123-134.

Chassaing K., Lasne N., Le Clainche C., Waser A.-M. (2011), «Travailler avec un cancer. Regards croisés sur les dispositifs d'aménagement des conditions de travail et sur les ressources mobilisées pour tenir ensemble travail et santé», Rapport de recherche, $\mathrm{n}^{\circ}$ 63, Noisy-leGrand, Centre d'études de l'emploi.

Chauvel L. (2001), «Le retour des classes sociales?», Revue de l'OFCE, $\mathrm{n}^{\circ}$ 79, pp. 315-359.

Chenu A. (2002), «Les horaires et l'organisation du temps de travail», Économie et statistique, n $352-353$, pp. 151-167.

Coutrot T., WolfF L. (2005), «L'impact des conditions de travail sur la santé : une expérience méthodologique», Rapport de recherche, $\mathrm{n}^{\circ} 23$, Noisy-le-Grand, Centre d'études de l'emploi.

DeSalvo K.B., Fan V.S., McDonnell M.B., Finh S.D. (2005), «Predicting mortality and healthcare utilization with a single question», Health services research, vol. $40, \mathrm{n}^{\circ} 4$, pp. 1234-1246.

Domens J., Pignier J. (2012), «Auto-entrepreneurs : au bout de trois ans, $90 \%$ dégagent un revenu inférieur au Smic au titre de leur activité non salariée», Insee première, $\mathrm{n}^{\circ} 1414$.

Eichenbaum-Voline S., Malavolti L., Paraponaris A., Ventelou B. (2008), «Cancer et activité professionnelle», Revue de l'OFCE, vol. 104, n 1, pp. 105-134.

Erdogan-Ciftci E., Van Doorslaer E., Bago D’Uva T., VAN LENTHE F. (2010), «Do self-perceived health changes predict longevity?», Social science \& medicine, vol. 71, $\mathrm{n}^{\circ} 11$, pp. 1981-1988.

Evain F., Amar M. (2006), «Les indépendants», Insee première, $\mathrm{n}^{\circ} 1084$.

Gollac M., Volkoff S. (2000), Les conditions de travail, Paris, La Découverte, coll. «Repères ».

Gollac M., Volkoff S. (2010), «Mesurer le travail. Une contribution à l'histoire des enquêtes françaises dans ce domaine», Document de travail, $\mathrm{n}^{\circ} 127$, Noisy-le-Grand, Centre d'études de l'emploi.

Gollac S. (2005), «Faire ses partages», Terrain, $n^{\circ} 45$, pp. 113-124.

INSEE (2009), Les revenus d'activité des indépendants, coll. «Insee Références».

InSERm (2011), Stress au travail et santé. Situation chez les indépendants, Paris, Inserm.

Jusot F., Khlat M., Sermet C. (2006), «Une mauvaise santé augmente fortement les risques de perte d'emploi», in Données sociales. La société française, Paris, Insee, pp. 533-542.

Khlat M., Sermet C. (2004), «La santé des chômeurs en France : revue de littérature», Revue d'épidémiologie et de santé publique, vol. 52, $\mathrm{n}^{\circ}$ 5, pp. 466-474.

Levratto N., Serverin É. (2009), «Être entrepreneur de soi-même après la loi du 4 août 2008 : les impasses d'un modèle productif individuel», Revue internationale de droit économique, t. XXIII, n 3 , pp. 325-352. 
Malenfant R., Larue A., Jetté M., Vézina M., St-Arnaud L. (2004), Précarisation du travail et santé : briser le cercle vicieux de l'exclusion, Ripost, CLSC Haute-Ville-Des-Rivières.

Maurin M.-L., Dupuy Y., Larré F., Sublet S. (éd.) (1999), «Prestation de travail et activité de service», Cahier travail et emploi, $\mathrm{n}^{\circ} 1999$.

Mesrine A. (2000), «La surmortalité des chômeurs : un effet catalyseur du chômage?», Économie et statistique, $n^{\circ} 334$, pp. 33-48.

Millunpalo S., Oja P., Pasanin M., Urponen H. (1997), «Self-rated health status as a health measure. The predictive value of self-reported health status on use of physician services and on mortality in the working age population », Journal of Clinical Epidemiology, vol. 50, $\mathrm{n}^{\circ} 5$, pp. 517-528.

Missègue N. (2000), «Le temps de travail des indépendants », Insee première, $\mathrm{n}^{\circ} 695$.

Perronnin M., Pierre A., Rochereau T. (2011), «La complémentaire santé en France en 2008 : une large diffusion, mais des inégalités d'accès », Questions d'économie de la santé, $\mathrm{n}^{\circ} 161$.

Ricroch L., Roumier B. (2011), «Depuis 11 ans, moins de tâches ménagères, plus d'Internet», Insee première, $\mathrm{n}^{\circ} 1377$.
RIESCO A. (2012), «Le travail indépendant des immigrés et sa sociologie», in Desmarez P., Lanciano-Morandat C., Monchatre S. et al., Temps, travail et salariat: Mateo Alaluf et Pierre Rolle, actualité de leur pensée, Toulouse, Octarès, pp. 57-66.

Rolle P. (1988), Bilan de la sociologie du travail, Tome 1, Grenoble, Presses universitaire de Grenoble.

Saurel-Cubizolles M.-J. (2001), «État de santé perçu et perte d'emploi», in Travail, santé, vieillissement, relations et évolutions : colloque des 18 et 19 novembre 1999, Toulouse, Octarès.

Spelten E.R., Sprangers M.A., Verbeek J.H. (2002), «Factors reported to influence the return to work of cancer survivors : a literature review », Psycho-Oncology, vol. $11, \mathrm{n}^{\circ} 2$, pp. 124-131.

Supiot A. (2009), Le droit du travail, Paris, Presses universitaires de France, coll. «Que sais-je?», 4e éd. mise à jour.

TAButEAu D. (2010), «La métamorphose silencieuse des assurances maladie», Droit social, n ${ }^{\circ}$, pp. 85-92.

Tubeuf S. (2010), «Mesure des inégalités de santé : la méthode "Ecuity" appliquée à la France», La Lettre du Collège des économistes de la santé, vol. $21, \mathrm{n}^{\circ} 1$, pp. 5-6. 